\title{
Study of high density phase transition in lattice QCD with canonical approach
}

\author{
Atsushi Nakamura \\ RIISE, Hiroshima University \\ Higashi-Hiroshima 739-8521, Japan \\ E-mail: hakamuradriise.hiroshima-u.ac.ip
}

\section{Shotaro Oka}

Institute of Theoretical Physics, Department of Physics, Rikkyo University

Toshima-ku, Tokyo 171-8501, Japan

E-mail: Ekasho-hatodrikkyo.ac.ip

\section{Asobu Suzuki}

Graduate School of Pure and Applied Sciences, University of Tsukuba

Tsukuba, Ibaraki 305-8571, Japan

E-mail: suzukichet.ph.tsukuba.ac.jp

\section{Yusuke Taniguchi*}

Graduate School of Pure and Applied Sciences, University of Tsukuba

Tsukuba, Ibaraki 305-8571, Japan

E-mail: tanigchiehet.ph.tsukuba.ac.jp

It is a well known fact that the finite density QCD system is suffered from the complex action problem. We apply the canonical apronch as a solution of the problem. We calculate observables like the pressure, the quark number density, its second cumulant and the chiral condensate as a function of the real chemical potential. We covered a wide range of temperature region starting from the confining low to the deconfining high temperature; $0.65 T_{c} \leq T \leq 3.62 T_{c}$. We see a possible signal of the deconfinement and the chiral restoration phase transition at real chemical potential below $T_{C}$ starting from the confining phase.

The 33rd International Symposium on Lattice Field Theory

14 -18 July 2015

Kobe International Conference Center, Kobe, Japan*

\footnotetext{
${ }^{*}$ Speaker.
} 


\section{Introduction}

The finite density QCD is a fascinating area from a physical point of view. The numerical lattice simulation should be a powerful tool to attack it. However, due to the notorious complex action problem its validity is limited. The fermion determinant $\operatorname{Det} D_{W}(\mu)$ becomes complex at real chemical potential $\mu$ and a naive Monte Carlo simulation does not work. One of the solution

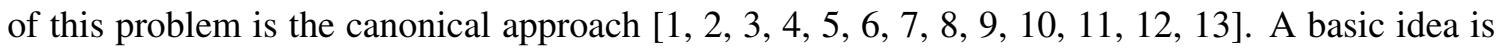
that the canonical partition function $Z_{C}(n, T, V)$ at a fixed quark number $n$ is related to the grand canonical one $Z_{G}(\mu, T, V)$ through a Fourier transformation

$$
Z_{C}(n, T, V)=\int_{0}^{2 \pi} \frac{d \frac{\mu_{I}}{T}}{2 \pi} e^{-i n \frac{\mu_{I}}{T}} Z_{G}\left(i \mu_{I}, T, V\right) .
$$

The point is that it is enough to calculate the grand canonical partition function at a pure imaginary chemical potential $i \mu_{I}$ in order to get the canonical one. So we escape from the complex action problem. One of the problem of the canonical approach is that the Dirac determinant needs to be evaluated accurately and frequently in $\mu_{I}$, which makes the computation heavy. A simple solution is to perform the fugacity expansion directly for the partition function

$$
Z_{G}(\mu, T, V)=\sum_{n=-\infty}^{\infty} Z_{C}(n, T, V) \xi^{n}, \quad \xi=e^{\mu / T}
$$

Fortunately it is possible to realize the fugacity expansion by a method of the temporal winding

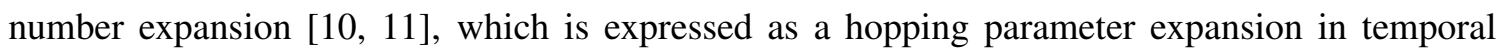
direction. By using the hopping parameter expansion the Dirac determinant can be evaluated with low cost and we can visit a wide parameter space in $\beta$ which corresponds to $0.65 T_{c} \leq T \leq 3.62 T_{c}$. The canonical partition function is evaluated for $N_{f}=2$ QCD both in the deconfinement and the confinement temperature regions. After derivation of the canonical partition function we study the chemical potential dependence of observables like the pressure, the quark number density and the chiral condensate. We see a possible signal of the confinement-deconfinement and chiral restoration phase transition at real chemical potential below the critical temperature $T_{c}$. A part of the results has been reported in Ref. [44, [5]]. For a detailed explanation of the numerical method to calculate the canonical partition function $Z_{C}(n, T, V)$ please see Ref. [ए5]].

\section{Canonical and grand canonical partition function}

We adopt the Iwasaki gauge action and the improved Wilson fermion action with the clover term. The clover coefficient is fixed to $c_{\mathrm{SW}}=1.1$ The APE stout smeared gauge link is used for those in the fermion action including the clover term. We adopt $8^{3} \times 4$ lattice. A wide range of $\beta$ is covered from high temperature $\beta=2.1$ to low temperature 0.9. A rough estimate of the temperature is given in a legend of each figure. The hopping parameter is selected in order that the hopping parameter expansion works well, for which $m_{\pi} / m_{\rho}$ turns out to be $0.7-0.9$. Maximal order of the hopping parameter expansion is set to 480 so that max winding number in temporal direction is 120 .

The first numerical result we get is the canonical partition function $Z_{C}(n, T, V)$. We plot $\log \left|Z_{C}(n, T, V) / Z_{C}(0, T, V)\right| /\left(V T^{3}\right)$ as a function of the quark number $n$ in Fig. $\mathbb{W}$. Data is shown 


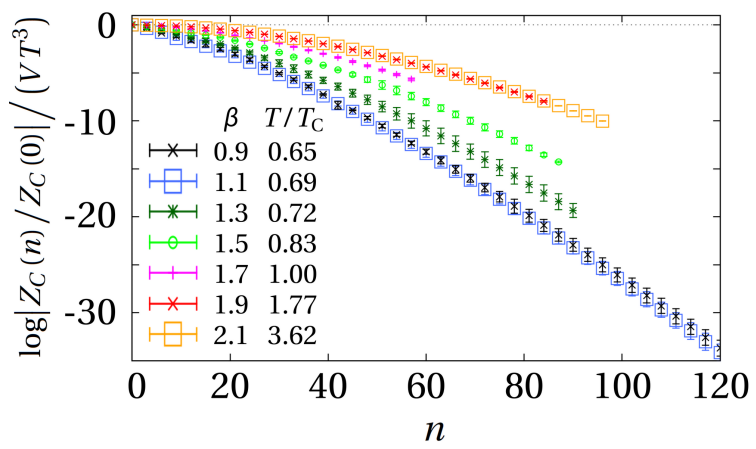

Figure 1: The canonical partition function $\log \left|Z_{C}(n) / Z_{C}(0)\right| /\left(V T^{3}\right)$ as a function of the quark number $n$. From the top $T / T_{c}=3.62$ (orange), 1.77 (red), 1.0 (magenta), 0.83 (green), 0.72 (dark green), 0.69 (blue) and 0.65 (black).

only for quark number $n$ which is multiple of three. The residual data do not contribute to the fugacity expansion (나) because of the $Z_{3}$ symmetry. The partition function decays very rapidly with $n$ and its behavior changes drastically between $T / T_{c}=1.77$ (red) and 0.83 (green). The data above $T / T_{c}=1.77$ is fitted very well by a quadratic function. But this is not the case below $T / T_{c}=0.83$.

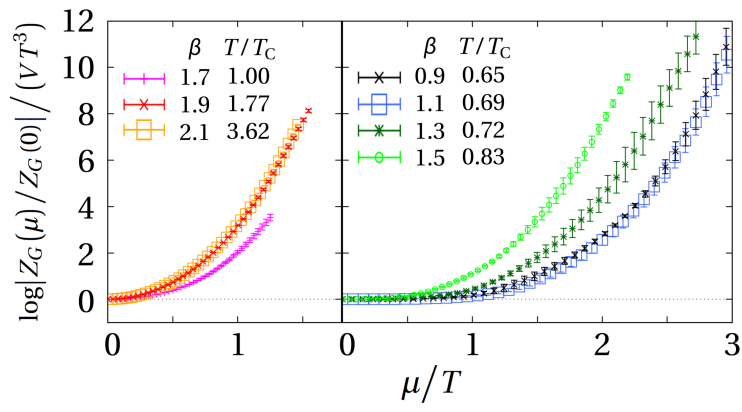

Figure 2: The grand partition function $\log \left|Z_{G}(\mu, T, V) / Z_{G}(0, T, V)\right| /\left(V T^{3}\right)$ as a function of the quark chemical potential $\mu / T$. The pressure at $T / T_{c}=0.65$ and 0.69 (right panel) are almost overlapped, and also $T / T_{c}=1.77$ and 3.62 (left panel) are completely overlapped.

The second physical quantity is the grand partition function. By taking summation for $-n_{\max } \leq$ $n \leq n_{\max }$ in equation (ㅁ.2) with $n$ multiple of three, we get the grand partition function for the real chemical potential. Here we notice that the canonical partition function $Z_{C}(n, T, V)$ is a real positive quantity if a Hermitian transfer matrix exists for the lattice QCD. However because of lattice artifact and numerical errors, they may deviate from real number. On the other hand we observed that the absolute value of the partition function is very stable against the statistical fluctuation and we take it as a tentative remedy. So instead of equation (ㄷ.2) we adopt

$$
Z_{G}(\mu, T, V)=\sum_{n=-n_{\max }}^{n_{\max }}\left|Z_{C}(n, T, V)\right| \xi^{n}
$$

for a definition of the grand partition function. Here we notice integers divisible by three contribute to the summation because of the $Z_{3}$ symmetry. From the partition function we get the pressure in 
the grand canonical ensemble

$$
\frac{1}{T^{4}}(P(\mu / T)-P(0))=\frac{1}{V T^{3}} \log \left|\frac{Z_{G}(\mu, T, V)}{Z_{G}(0, T, V)}\right| .
$$

We plot the pressure normalized at $\mu=0$ in Fig. $\square$ as a function of the quark chemical potential. We observe that the pressure is very small at small chemical potential for low temperature below $T_{c}$ as is shown in the left panels of Fig. \.

\section{Quark number and its second cumulant}

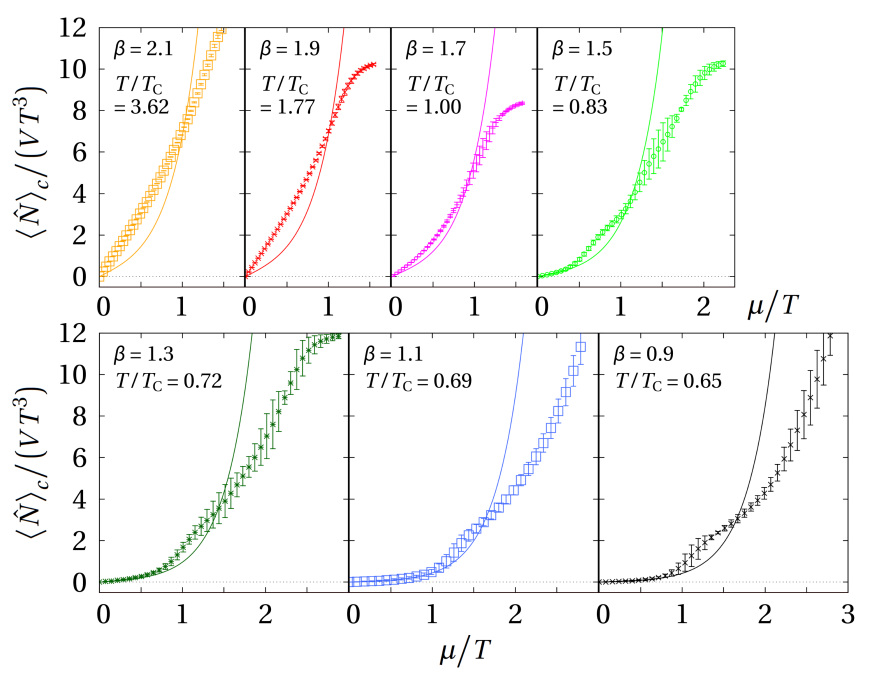

Figure 3: Quark number density $\langle\hat{N}\rangle /\left(V T^{3}\right)$ as a function of the quark chemical potential.

The third physical application is the $k$-th moment of the quark number operator

$$
\left\langle\hat{N}^{k}\right\rangle=\frac{1}{Z_{G}(\mu, T, V)} \sum_{n=-n_{\max }}^{n_{\max }} n^{k}\left|Z_{C}(n, T, V)\right| \xi^{n} .
$$

In Fig. B, the quark number density $\langle\hat{N}\rangle /\left(V T^{3}\right)$ is plotted as a function of the real quark chemical potential $\mu / T$. The data below $T_{c}$ is consistent with the hadron resonance gas prediction $\langle\hat{N}\rangle /\left(V T^{3}\right) \propto$ $\sinh \left(3 \frac{\mu}{T}\right)$ as is indicated by the solid line. This property is also understandable as a leading order contribution from the fugacity expansion (B.D)

$$
\frac{\langle\hat{N}\rangle}{\left(V T^{3}\right)}=\frac{1}{V T^{3}} \frac{6\left|Z_{C}(3, T, V)\right|}{\left|Z_{C}(0, T, V)\right|} \sinh \left(3 \frac{\mu}{T}\right)+\mathscr{O}\left(Z_{C}(6, V, T), Z_{C}(3, T, V)^{2}\right) .
$$

The solid line in Fig. B is a plot of this contribution. The data is well described with (B.2) for $T<T_{c}$ since $Z_{C}(n, T, V)$ decays rapidly with $n$ as is shown in Fig $\mathbb{U}$. The sinh function is not a good description for $T \geq T_{c}$. The number density rises linearly with $\mu / T$ near the origin as is indicated by the free quark gas model

The second cumulant of the quark number density may be a good indicator of a phase transition. The results are plotted in Fig. 田. We observe peaks around $\mu / T \sim 1$ for every $\beta$. We also 

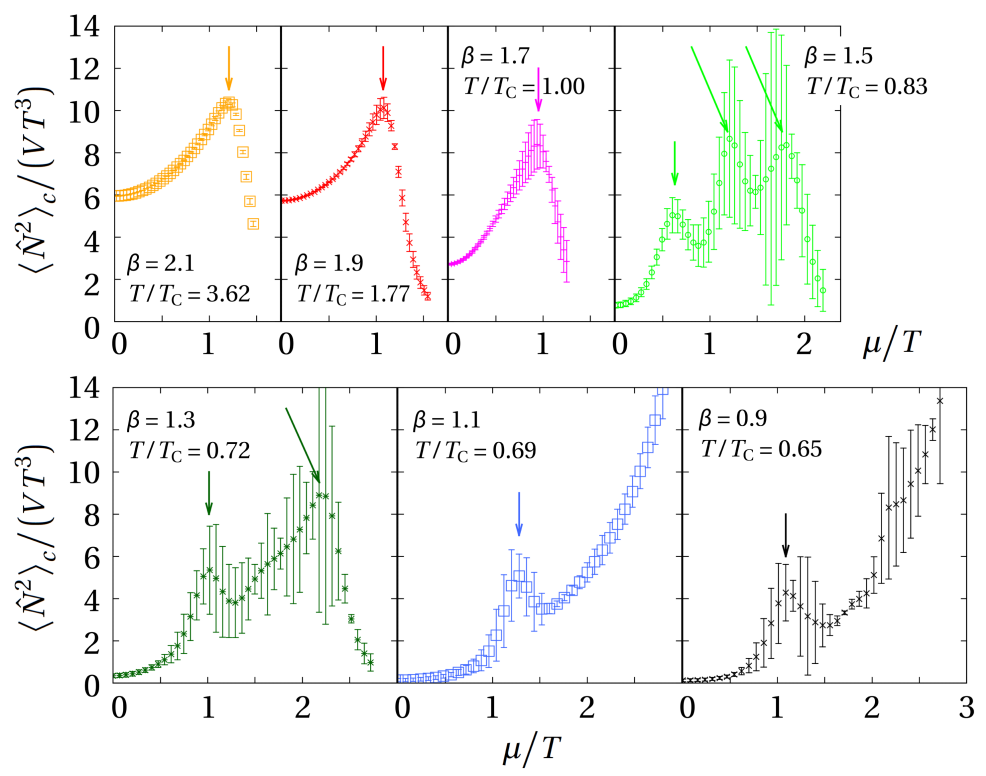

Figure 4: The second cumulant of the quark number $\left\langle\hat{N}^{2}\right\rangle_{c} /\left(V T^{3}\right)$ as a function of the quark chemical potential. The lower panels plot results for $T / T_{c}=0.65,0.69$ and 0.72 below $T_{c}$. The upper panel is results for $T_{c}=0.83-3.62$.

observe secondary peaks for $T / T_{c}=0.72$ (dark-green) and 0.83 (green) at larger $\mu / T$, while only a single peak is observed at high temperature $T \geq T_{c}$. Although the secondary peaks are not shown for $T / T_{c}=0.65$ and 0.69 they exist at $\mu / T>3$. These peaks show a distinctive behavior against the quark number cut-off $n_{\max }$. The first peaks below $T_{c}$ are stable even if we vary $n_{\max }$ by $20 \%$. It would be conjectured that the first peaks in the low temperature side are the physical indication of the confinement-deconfinement phase transition. Unfortunately we cannot exclude a possibility that these first peak corresponds to an onset phenomenon. We need a further study at lighter quark mass to have a firm conclusion. The second peaks at $T / T_{c}=0.72,0.83$ and the first peaks above $T_{c}$ change their height and position with $n_{\max }$ changes. These peaks would be artifacts due to the cut-off in quark number in the fugacity expansion.

The position of the first peak below $T_{c}$ is consistent with a physical expectation that the position moves to larger $\mu / T_{c}$ as temperature decreases. We plot a rough estimate of the peak position in $\mu-T$ plane in Fig. [5. We set $\mu_{c}=0$ at $T=T_{c}$. We notice this is a preliminary plot since the quark mass is not the same between different $\beta$ and no error estimation is performed. We drop the data for $T / T_{c}=0.65$ since the lattice artifact seems to be very large in the strong coupling region.

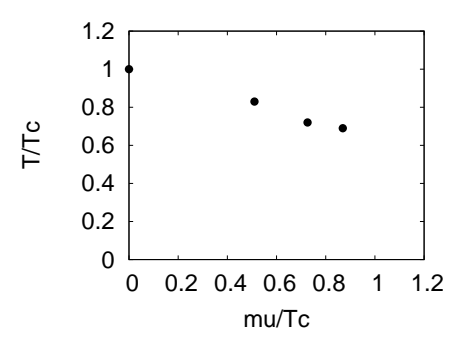

Figure 5: A rough estimate of the first peak of the second cumulant below $T_{c}$ and its plot in the $\mu-T$ plane. No error estimation is performed. 


\section{Chiral condensate}

We apply the fugacity expansion both to numerator and denominator of a hadronic VEV

$$
\langle O\rangle_{G}(\mu, T, V)=\frac{O_{G}(\mu, T, V)}{Z_{G}(\mu, T, V)}, \quad O_{G}(\mu, T, V)=\sum_{n=-\infty}^{\infty} O_{C}(n, T, V) \xi^{n}
$$

Once we have two coefficients $Z_{C}(n, T, V)$ and $O_{C}(n, T, V)$, the VEVs of the operator in the canonical and the grand canonical ensemble are available. The chiral condensate $-\int d^{3} x\langle\bar{\psi} \psi\rangle_{C} /\left(V T^{3}\right)$ in canonical ensemble is given in the left panel of Fig. 6 as a function of the quark number. That in the grand canonical ensemble is given in the right panel of Fig. 6 as a function of the real chemical potential $\mu / T$. The condensate in the figure is a bare quantity without renormalization. Since we adopted the Wilson fermion we have an additive correction, which is not subtracted. From the fig-
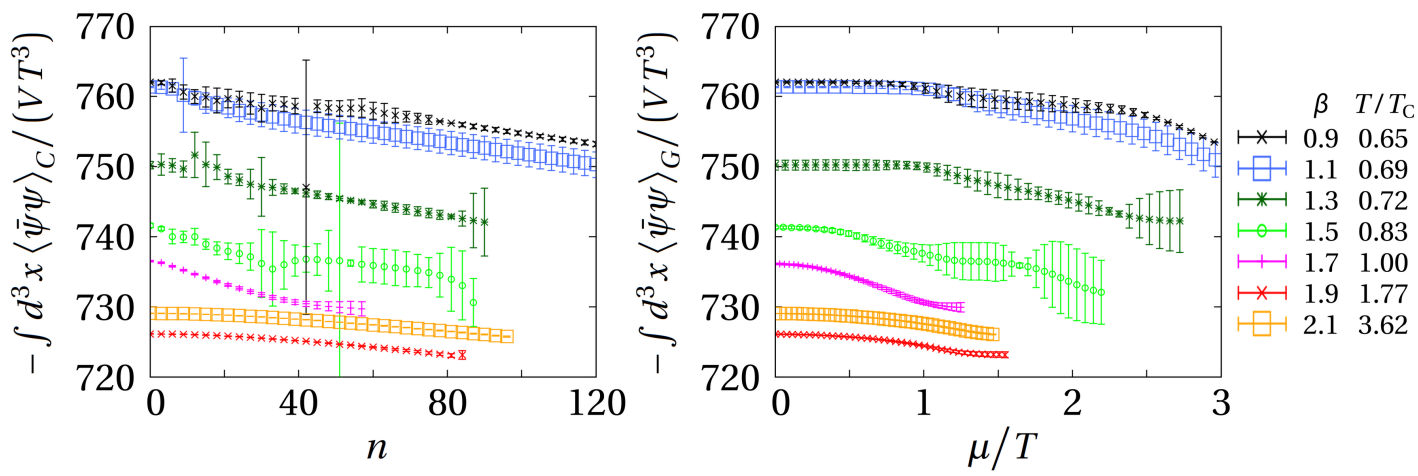

Figure 6: The chiral condensate $-\int d^{3} x\langle\bar{\psi} \psi\rangle /\left(V T^{3}\right)$ in the canonical ensemble (left panel) as a function of the quark number. The right panel is that in the grand canonical ensemble as a function of the quark chemical potential $\mu / T$. The color and $\beta$ correspondence is the same as in Fig.

ure, the chiral restoration phase transition at finite chemical potential seems to be seen. A relatively large value at around $\mu / T=0$ starts to decrease rapidly at chemical potential $\mu / T \sim 1$ for low temperature. The would-be transition parameter $\mu_{c} / T$ becomes larger for lower temperature and smaller for high temperature although we need to notice the quark mass is not the same for each $\beta$. The position of $\mu_{c} / T$ seems to be consistent with that of the first peak for the second cumulant in Fig. 团 below $T_{c}$.

\section{Discussion}

We adopt the canonical approach as a solution of the complex action problem in finite density QCD. We calculate the canonical partition function $Z_{C}(n, T, V)$ and extract observables like the pressure, the quark number density, its second cumulant and the chiral condensate as a function of the real chemical potential. We see a possible signal of the deconfinement and the chiral restoration phase transition. 
In general the complex action problem turns out to be the sign problem when we try to solve it. In the canonical approach the sign problem may remain in the complex phase of the canonical partition function. Although it should be real and positive the corresponding operator has a complex phase for each gauge configuration. A scattered plot of the observable for $Z_{C}(n=3)$ is given in Fig. $\square$. Notice that we take a $\log$ for its absolute value. A fluctuation in the complex phase is tiny for $T>T_{c}$ as is shown by the red symbol. However it becomes severe for $T \leq T_{c}$ as can be seen from the magenta and green symbols. This is the problem we

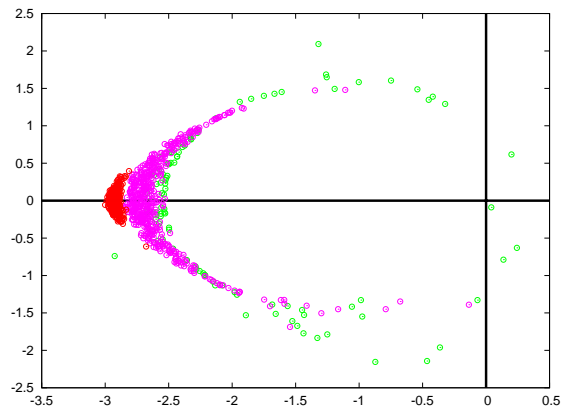

Figure 7: A scattered plot of $\log \mid Z_{C}(n=$ 3) $\mid e^{i \theta}$ for $T / T_{c}=1.77$ (red), 1.0 (magenta) and 0.83 (green).

need to solve in future.

This work is done for $\mathrm{Zn}$ Collaboration. This work is supported in part by Grants-in-Aid of the Ministry of Education (Nos. 26610072, 24340054, 22540265, 15H03663, 26610072). This work is in part based on Lattice QCD common code Bridge++ [ए6]. This work is supported by the Large Scale Simulation Program No.14/15-19 (FY2014) of High Energy Accelerator Research Organization (KEK).

\section{References}

[1] A. Hasenfratz and D. Toussaint, Nucl. Phys. B 371, 539 (1992).

[2] S. Kratochvila and P. de Forcrand, Nucl. Phys. Proc. Suppl. 140, 514 (2005).

[3] P. de Forcrand and S. Kratochvila, Nucl. Phys. Proc. Suppl. 153, 62 (2006).

[4] A. Borici, Prog. Theor. Phys. Suppl. 153, 335 (2004).

[5] K. Nagata and A. Nakamura, Phys. Rev. D 82, 094027 (2010).

[6] A. Alexandru and U. Wenger, Phys. Rev. D 83, 034502 (2011).

[7] A. Li, A. Alexandru and K. F. Liu, Phys. Rev. D 84, 071503 (2011).

[8] K. Nagata et al. [XQCD-J Collaboration], PTEP 2012, 01 A103 (2012).

[9] A. Alexandru, M. Faber, I. Horvath and K. F. Liu, Phys. Rev. D 72, 114513 (2005).

[10] X. f. Meng, A. Li, A. Alexandru and K. F. Liu, PoS LATTICE 2008, 032 (2008).

[11] A. Li, A. Alexandru, K. F. Liu and X. Meng, Phys. Rev. D 82, 054502 (2010).

[12] J. Danzer and C. Gattringer, Phys. Rev. D 86, 014502 (2012).

[13] C. Gattringer and H. P. Schadler, arXiv:1411.5133 [hep-lat].

[14] A. Nakamura, S. Oka and Y. Taniguchi, PoS LATTICE 2014, 198 (2015).

[15] A. Nakamura, S. Oka and Y. Taniguchi, arXiv:1504.04471 [hep-lat].

[16] http://bridge.kek.jp/Lattice-code/index_e.html 